\title{
Will my patient survive? Look for creatinine in the urine!
}

\author{
Michael Darmon ${ }^{1,2,3^{*}} \mathbb{D}$, Kianoush Kashani ${ }^{4,5}$ and Miet Schetz ${ }^{6}$
}

(0) 2018 Springer-Verlag GmbH Germany, part of Springer Nature and ESICM

The ability to risk stratify critically ill patients and target intensive care unit resources to those who benefit the most is appealing and has resulted in a growing interest in the search for novel risk factors [1, 2]. Performance status has gained significant attention recently $[3,4]$. In the same line, poor nutritional status, often judged by a low body mass index (BMI), is a prominent factor associated with poor short- and long-term outcomes $[5,6]$. Several assessments of nutritional status and muscle mass have been evaluated and validated, such as albumin and prealbumin levels, anthropometric measurements, skeletal muscle mass or fat infiltration measured by imaging studies, various scores or multicomponent scales, fatfree mass or the sarcopenia index [7-9]. Some of these parameters have been reported to be associated with outcome in ICU patients [7-9].

In the steady state, creatinine is produced by skeletal muscle cells and excreted in the urine at a relatively constant rate. Timed measurement of urinary creatinine excretion (UCE) could be, therefore, a valid reflection of muscle mass and has been associated with survival in patients with coronary artery disease [10-12]. In an article recently published in Intensive Care Medicine, Hessels et al. reported the performance of $24 \mathrm{~h}$ UCE in the prediction of short- and long-term outcome in ICU patients [13]. This retrospective cohort study included patients $\geq 15$ years old, admitted to a single center between 2002 and 2015, and in whom $24 \mathrm{~h}$ urinary creatinine was measured during the first 3 days of admission. The study outcomes included the hospital and 5-year mortality rates. Among the 37283 patients admitted

*Correspondence: michael.darmon@aphp.fr

${ }^{1}$ Medical ICU, Saint-Louis University Hospital, AP-HP, 1 Avenue Claude Vellefaux, 75010 Paris, France

Full author information is available at the end of the article during the study period, 6151 met the eligibility criteria. UCE in $24 \mathrm{~h}$, before and after adjustment for confounders [i.e., age, BMI, estimated glomerular filtration rate (eGFR), illness severity and trauma] and stratified for gender, was found to be an independent predictor of outcome (i.e., low urinary creatinine excretion was associated with higher short- and long-term mortality). Several subgroups and sensitivity analyses performed on patients according to age, gender, BMI, underlying acute kidney injury, and trauma as a reason for ICU admission or presence of rhabdomyolysis, confirmed the main findings.

The authors have to be congratulated for this interesting study. It confirms that a simple and non-expensive measurement of urine creatinine excretion, as a surrogate of muscle mass, provides important prognostic information and may add to the existing prognostic scores.

There are also several limitations to this study which need to be underlined. First, creatinine excretion only reflects production (muscle mass) when GFR is in steady state. This assumption is probably not valid in ICU patients during the first 3 days. In addition, the authors used eGFR by the CKF-EPI equation to adjust for kidney function, which, in view of the delay of serum creatinine in detecting changes in GFR and the variability in serum creatinine due to factors unrelated to kidney function (such as fluid overload, decreased creatinine production related to low muscle mass or sepsis), is an imperfect reflection of true GFR [14, 15]. Hence, the assumption that urinary creatinine excretion solely reflects muscle creatinine production is not verifiable. More importantly, the reported finding that urinary creatinine excretion was associated with the clinical outcome could be related to the influence of underlying AKI which could lower urinary creatinine excretion per se. Lastly, serum creatinine is not sensitive enough to detect small day-by-day changes in GFR. The authors partially addressed this limitation by excluding patients with stage 3 AKI and

\section{6 Springer}




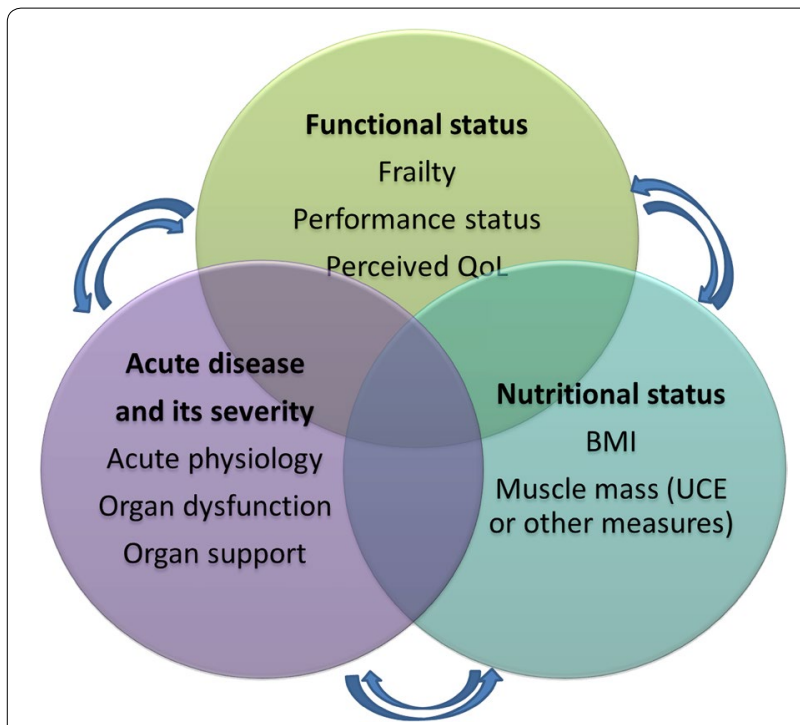

Fig. 1 Intrication between functional status, nutritional status and acute disease

by using the median UCE over the first 3 days for each patient. In addition, mean UCE was similar on day 1 and 3 , and the results were similar with estimated UCE accounting for the change in serum creatinine. The effect was observed in patients with and without AKI, and the adjustment for kidney function yielded similar results with the eGFR or measured creatinine clearance (a better reflection of true GFR).

Second, the findings are not externally validated, and; hence, the results may not be generalizable. The vast majority of patients admitted during the study period were excluded for ICU stay less than $24 \mathrm{~h}$ or missing creatinine excretion. This study was performed in a single center, and factors such as overall BMI or overall nutritional status may reflect factors specific to the study center [16]. Ethnicity, another determinant of muscle mass, was not reported. The adjustment also does not account for comorbidities and frailty. Although sarcopenia, frailty, and comorbidities are partly correlated, these three preexisting conditions are distinct events, and each of them may be associated with outcome [17].

And finally, even though this study gives reliable clues suggesting that creatinine excretion might be independently associated with outcome, its relevance to the clinical practice is debatable. Optimal nutrition strategies in ICU remain controversial, the influence of early mobilization is likely to extend across nutritional strata, and significance of the findings at an individual patient versus population level is uncertain.

Despite these limitations, the study by Hessels et al. certainly raises several hypotheses requiring additional studies. Validation of these findings in larger multicenter studies, ideally across several countries and continents, may increase its generalizability. These subsequent studies should ideally include an adequate adjustment for real-time changes in renal function to better discriminate the role of GFR changes from the role of sarcopenia. Ideally, multiple techniques to assess nutritional status/sarcopenia should be compared, with a clear description of the test diagnostic or prognostic performance, including the rate of misclassification and predictive model calibration. Such studies will also help in delineating the ideal measure of nutritional status at the bedside while taking into account confounders both at patient and population levels (Fig. 1).

\section{Author details \\ ${ }^{1}$ Medical ICU, Saint-Louis University Hospital, AP-HP, 1 Avenue Claude Vellefaux, 75010 Paris, France. ${ }^{2}$ Faculté de Médecine, Université Paris-Diderot, Sorbonne-Paris-Cité, Paris, France. ${ }^{3}$ ECSTRA team, Biostatistics and clinical epidemiology, UMR 1153 (center of epidemiology and biostatistics Sorbonne Paris Cité, CRESS), INSERM, Paris, France. ${ }^{4}$ Division of Nephrology and Hyper- tension, Department of Medicine, Mayo Clinic, Rochester, MN, USA. ${ }^{5}$ Division of Pulmonary and Critical Care Medicine, Department of Medicine, Mayo Clinic, Rochester, MN, USA. ${ }^{6}$ Clinical Department and Laboratory of Intensive Care Medicine, Division of Cellular and Molecular Medicine, KU Leuven Univer- sity, Herestraat 49, B3000 Louvain, Belgium.}

Funding

None.

Compliance with ethical standards

\section{Conflicts of interest}

The other authors declare having no conflict of interest related to this manuscript.

\section{Ethical approval}

Not applicable.

Received: 24 September 2018 Accepted: 28 September 2018 Published online: 4 October 2018

\section{References}

1. Pirracchio R, Petersen ML, Carone M et al (2015) Mortality prediction in intensive care units with the Super ICU learner algorithm (SICULA): a population-based study. Lancet Respir Med 3:42-52. https://doi.org/10.1016/ S2213-2600(14)70239-5

2. Muller $\mathrm{G}$, Flecher E, Lebreton $\mathrm{G}$ et al (2016) The ENCOURAGE mortality risk score and analysis of long-term outcomes after VA-ECMO for acute myocardial infarction with cardiogenic shock. Intensive Care Med 42:370-378. https://doi.org/10.1007/s00134-016-4223-9

3. Zampieri FG, Iwashyna TJ, Viglianti EM et al (2018) Association of frailty with short-term outcomes, organ support and resource use in critically ill patients. Intensive Care Med 44:1512-1520. https://doi.org/10.1007/ s00134-018-5342-2

4. Zampieri FG, Bozza FA, Moralez GM et al (2017) The effects of performance status one week before hospital admission on the outcomes of critically ill patients. Intensive Care Med 43:39-47. https://doi. org/10.1007/s00134-016-4563-5 
5. Hutagalung R, Marques J, Kobylka K et al (2011) The obesity paradox in surgical intensive care unit patients. Intensive Care Med 37:1793-1799. https://doi.org/10.1007/s00134-011-2321-2

6. Mogensen KM, Robinson MK, Casey JD et al (2015) Nutritional status and mortality in the critically ill. Crit Care Med 43:2605-2615. https://doi. org/10.1097/CCM.0000000000001306

7. Nishikawa H, Osaki Y (2015) Liver cirrhosis: evaluation, nutritional status, and prognosis. Mediators Inflamm. https://doi.org/10.1155/2015/872152

8. Thibault R, Makhlouf A-M, Mulliez A et al (2016) Fat-free mass at admission predicts 28-day mortality in intensive care unit patients: the international prospective observational study phase angle project. Intensive Care Med 42:1445-1453. https://doi.org/10.1007/s00134-016-4468-3

9. Kashani KB, Frazee EN, Kukrálová L et al (2017) Evaluating muscle mass by using markers of kidney function: development of the sarcopenia index. Crit Care Med 45:e23-e29. https://doi.org/10.1097/CCM.0000000000 002013

10. Heymsfield SB, Arteaga C, McManus C et al (1983) Measurement of muscle mass in humans: validity of the 24-hour urinary creatinine method. Am J Clin Nutr 37:478-494. https://doi.org/10.1093/ajcn/37.3.478

11. Pirlich M, Selberg O, Böker K et al (1996) The creatinine approach to estimate skeletal muscle mass in patients with cirrhosis. Hepatol Baltim Md 24:1422-1427. https://doi.org/10.1002/hep.510240620

12. Ix JH, de Boer IH, Wassel CL et al (2010) Urinary creatinine excretion rate and mortality in persons with coronary artery disease: the Heart and Soul
Study. Circulation 121:1295-1303. https://doi.org/10.1161/CIRCULATIO NAHA. 109.924266

13. Hessels L, Koopmans N, Gomes Neto AW et al (2018) Urinary creatinine excretion is related to short-term and long-term mortality in critically ill patients. Intensive Care Med. https://doi.org/10.1007/s00134-018-5359-6

14. Bragadottir G, Redfors B, Ricksten S-E (2013) Assessing glomerular filtration rate (GFR) in critically ill patients with acute kidney injury-true GFR versus urinary creatinine clearance and estimating equations. Crit Care 17:R108. https://doi.org/10.1186/cc12777

15. Ichai C, Vinsonneau C, Souweine B et al (2016) Acute kidney injury in the perioperative period and in intensive care units (excluding renal replacement therapies). Ann Intensive Care 6:48. https://doi.org/10.1186/s1361 3-016-0145-5

16. Worldwide trends in body-mass index, underweight, overweight, and obesity from 1975 to 2016: a pooled analysis of 2416 population-based measurement studies in 128.9 million children, adolescents, and adults. https://www.ncbi.nlm.nih.gov/pmc/articles/PMC5735219/. Accessed 11 Sep 2018

17. Reeve TE, Ur R, Craven TE et al (2018) Grip strength measurement for frailty assessment in patients with vascular disease and associations with comorbidity, cardiac risk, and sarcopenia. J Vasc Surg 67:1512-1520. https ://doi.org/10.1016/j.jvs.2017.08.078 